\title{
The clinical relevance of manometric esophagogastric junction outflow obstruction can be determined using rapid drink challenge and solid swallows
}

Short Title: Clinical relevance of EGJOO

Santosh Sanagapalli, Joshua McGuire, Rupert Leong, Kalp Patel, Amanda Raeburn, Humayra Abdul-Razakq, Andrew Plumb, Matthew Banks, Rehan Haidry, Laurence Lovat, Vinay Sehgal, David Graham, Sarmed S. Sami, Rami Sweis

GI Physiology Unit, University College London Hospital, 235 Euston Rd London, United Kingdom NW1 2BU

Corresponding author: Dr. S. Sanagapalli, email: Santosh.sanagapalli@svha.org.au

Word count: 2,997 words

Guarantor: Santosh Sanagapalli

Financial support: None provided

Competing interests: None to declare

Ethical considerations: This study was reviewed and approved by the Institutional Review Board of University College London Hospital.

Author contributions: Study conception and design: SSanagapalli, RS; Data acquisition: SSanagapalli, JM, RS, AR, HAR, KP, AP; Data analysis: SSanagapalli; Drafting of manuscript: SSanagapalli; Critical review of manuscript: SSanagapalli, RL, MB, RH, LL, VS, DG, SSami, RS 


\section{Study highlights}

\section{What is known}

- Esophagogastric junction outflow obstruction (EGJO0) is a manometric entity of unclear clinical significance

- Some cases represent an achalasia-like syndrome, but many are spurious findings, often improving without specific therapy

- There is no established method to differentiate the two

- Provocative testing during high resolution manometry, using rapid drink challenge and solid swallows, has been shown to improve the diagnostic accuracy for other motility disorders compared with the standard manometry protocol.

\section{What is new here}

- Provocative testing can differentiate EGJ00 cases that respond to therapy from those that remit spontaneously with a high degree of diagnostic accuracy

- Barium esophagography appears to miss many cases of clinically relevant EGJOO

- EGJOO with raised integrated relaxation pressure on $5 \mathrm{~mL}$ water swallows but not on provocative testing rarely represent clinically significant disease requiring therapy 


\section{Abstract}

\section{Objectives}

Esophagogastric junction outflow obstruction (EGJOO) defined on high-resolution manometry (HRM) poses a management dilemma given marked variability in clinical manifestations. We hypothesized that findings from provocative testing (rapid drink challenge and solid swallows) could determine clinical relevance of EGJOO.

\section{Methods}

In a retrospective cohort study, we included consecutive patients between May 2016 and January 2020 with EGJOO. Standard HRM with 5mL water swallows was followed by provocative testing. Barium esophagography findings were obtained. Cases with structural obstruction were separated from functional EGJOO, with the latter categorized as symptom-positive or symptom-negative. Only symptom-positive subjects were considered for achalasia-type therapies. Sensitivity and specificity for clinically relevant EGJOO during 5mL water swallows, provocative testing and barium was calculated.

\section{Results}

Of the 121 EGJOO cases, $76 \%$ had dysphagia and $25 \%$ had holdup on barium. Ninetyseven (84\%) were defined as functional EGJOO. Symptom-positive EGJOO subjects were more likely to demonstrate abnormal motility, pressurization patterns and to reproduce symptoms during provocative testing, but not with $5 \mathrm{~mL}$ water swallows. Twenty-nine (30\%) functional EGJOO patients underwent achalasia-type therapy, with symptomatic response in 26 (90\%). Forty-eight (49\%) functional EGJOO cases were managed conservatively, with symptom remission in $78 \%$. While specificity was similar, 
provocative testing demonstrated superior sensitivity in identifying treatment responders from spontaneously remitting EGJO0 (85\%) compared to both 5mL water swallows (54\%; $\mathrm{p}<0.01)$ and barium esophagography (54\%; $\mathrm{p}=0.02)$.

\section{Conclusions}

Provocative testing during HRM is highly accurate in identifying clinically relevant EGJOO that benefits from therapy and should be routinely performed as part of the manometric protocol.

Keywords: esophagogastric junction outflow obstruction, high-resolution manometry, rapid drink challenge, provocative testing, pneumatic dilatation 


\section{Introduction}

Esophagogastric junction outflow obstruction (EGJOO) is a manometric entity defined by the combination of raised integrated relaxation pressure (IRP) and intact peristalsis on high-resolution esophageal manometry (HRM). ${ }^{1}$ EGJOO poses a management dilemma because of significant heterogeneity in clinical manifestations. Many cases are minimally symptomatic, improve without specific therapy, and may sometimes represent manometric artefacts. ${ }^{2-4}$ Yet EGJOO can also resemble a variant of achalasia with marked symptoms, favorable response to achalasia-type therapies, and in some cases clear progression to achalasia on interval testing. ${ }^{5-7}$ Further, EGJOO can also be associated with structural pathologies at the EGJ.8,9

While the identification and management of those with structural causes of EGJOO is usually straightforward, the key clinical conundrum remains how to reliably identify clinically relevant cases of functional EGJOO. Confirming that the IRP remains raised in the upright position excludes some spurious cases, but not all. ${ }^{10,} 11$ Barium esophagography is a useful complementary test, 5,12 but can miss cases of clinically relevant EGJ00.13 Functional lumen imaging probe (FLIP) measurement of lower esophageal sphincter (LES) distensibility may be more accurate, ${ }^{13}$ but requires additional technology.

A potential explanation for the poor specificity of manometric EGJOO diagnoses may be the standard protocol, which uses small volume $5 \mathrm{~mL}$ water swallows in the supine position. It is self-evident that this is not reflective of normal eating and drinking, and there is inherent logic in performing motility testing using larger volumes of water and 
inclusion of solids. At our institution, such "provocative testing" is performed routinely during all HRM studies. Inclusion of the rapid drink challenge (RDC) and solid foods during HRM has been shown to increase the diagnostic yield for clinically relevant outflow obstruction in achalasia ${ }^{14}$ and post-fundoplication dysphagia, ${ }^{15}$ but their value in determining clinical relevance of functional EGJOO has not been studied.

This study aimed to compare the diagnostic accuracy of three modalities in identifying clinically relevant functional EGJO0; namely, HRM using 5mL swallows, HRM using provocative testing, and barium esophagography. In the absence of a reference standard, we aimed to determine relevance firstly using symptomatology at presentation, and secondly, the natural history of the disease process or therapeutic outcomes over time. We hypothesized that findings from RDC and solid swallows would be superior to other modalities in identifying clinically relevant, symptomatic EGJOO.

\section{Methods}

\section{Subjects}

A retrospective cohort study was performed. All HRM studies performed at single tertiary referral center between May 2016 and January 2020 were reviewed, and patients with a manometric finding of EGJOO were eligible for inclusion. This study was reviewed and approved by the hospital Institutional Review Board.

\section{High-resolution manometry}

HRM was performed using a 36-channel, solid-state system (Manoscan Eso Z, Medtronic, Hertfordshire, UK). In all patients, a standard protocol was performed whereby ten $5 \mathrm{~mL}$ 
water swallows were administered. Swallows were performed in the upright, seated position to replicate normal behavior as per our previously published standard. ${ }^{14,16-18}$ Subsequently, the RDC was performed whereby the patient was instructed to continuously drink $200 \mathrm{~mL}$ of water through a straw without stopping. Finally, solids were administered either in the form of five to ten $1 \mathrm{~cm}^{3}$ cubes of buttered white bread or a test meal of $200 \mathrm{~g}$ of white rice, depending on patient ability.

We extrapolated Chicago Classification criteria for EGJOO based on $5 \mathrm{~mL}$ swallows, to incorporate findings from RDC and solid swallows using established definitions for EGJ obstruction on provocative testing. ${ }^{16,}{ }^{19}$ Specifically, patients were defined as EGJOO when (i) IRP was raised, and (ii) there was evidence of intact peristalsis. ${ }^{1}$ IRP was considered raised when any of the following were present: median IRP above $15 \mathrm{mmHg}$ with $5 \mathrm{~mL}$ water swallows, ${ }^{1,20} 8 \mathrm{mmHg}$ during $\mathrm{RDC},{ }^{16}$ or $25 \mathrm{mmHg}$ for at least 2 solid swallows. $^{19,} 21$ The presence of any abnormalities of esophageal peristalsis, pressurization or reproduction of the patient's typical symptoms during any of the three modalities was recorded.

\section{Barium esophagography}

Timed or conventional barium swallows were undertaken. Timed barium swallows were performed in the standard manner whereby $200 \mathrm{~mL}$ is imbibed by the patient while standing, with images taken at one-, two- and five-minutes post-ingestion. ${ }^{22}$ A barium column of $1 \mathrm{~cm}$ or more in height above the EGJ at the one-minute mark was considered positive. On conventional barium swallow, any finding of barium holdup at the EGJ was considered positive. 


\section{Study design}

Endoscopy, histology and barium esophagography were assessed where available for each case. EGJOO was considered structural if an anatomical abnormality was identified at the EGJ on endoscopy or imaging that could explain the manometric finding. ; p Patients were defined as having functional EGJOO only when structural obstruction was excluded. Functional EGJOO was classified as "symptom-negative" if patients were minimally symptomatic (ES $\leq 3$ and dysphagia sub-score $\leq 1$ ), or if an alternative diagnosis was established that better explained the symptoms (e.g. cricopharyngeal stricture or functional gastrointestinal disorder). The remainder were considered "symptompositive" EGJOO.

Per convention, a reduction in ES to $\leq 3$ at three months following therapy determined treatment success. Any patient achieving this after primary or secondary therapy was considered a "responder". For mildly symptomatic patients (ES $\leq 3$ ) managed conservatively, we considered a reduction of ES by at least 1 point at follow up to be a successful response ("spontaneous remitters").

\section{Therapy}

Only symptom-positive functional EGJOO was considered amenable for achalasia-type therapy to the LES. However, the decision for therapy was also dependent on other factors e.g. comorbidities, other management options, willingness to withdraw opiates, patient preference. The decision and choice of therapy therefore came about after a discussion of benefits and risks with the patient. Therapeutic options included Botulinum toxin injection (100IU) into the LES, pneumatic dilatation using a graded regimen, ${ }^{23}$ and per-oral endoscopic myotomy (POEM). For EGJOO in opiate users, dose reduction or 
cessation was also considered. The Eckardt score (ES) was administered to all patients at baseline and at every follow-up.

\section{Analysis}

Firstly, diagnostic variables and outcomes were compared between symptom-positive and symptom-negative groups. Wilcoxon rank sum test or $t$-test were used as appropriate to compare continuous variables. Fisher's exact test was used for categorical data comparison. Secondly, McNemar's test was used to evaluate sensitivity and specificity of the three diagnostic modalities in identifying clinically relevant EGJ00. In the absence of a reference standard, we used responders and spontaneous remitters as surrogates for the presence or absence of disease respectively. For this analysis, $5 \mathrm{~mL}$ water swallows and provocative tests were considered positive if (i) they demonstrated a raised IRP, and (ii) they demonstrated any evidence of abnormal peristalsis, pressurization patterns or reproduction of typical symptoms. $P$ values of $<0.05$ were considered statistically significant. Data were analyzed using SPSS Statistics for Mac, version 23.0 (IBM Corp, Armonk, NY, USA).

\section{Results}

From a total of 1640 HRM studies performed during the study period, EGJOO was identified in 121 cases (7.4\%; Figure 1). Clinico-manometric characteristics are described in Table 1 . All 121 subjects completed ten $5 \mathrm{~mL}$ water swallows. Due to patient intolerance 112 (93\%) completed RDC and 116 (96\%) completed solid swallows; however, 119 (98\%) had at least one form of provocative test. Most subjects had raised IRP detected by multiple bolus types (Figure 2). Eleven subjects (9\%) had raised IRP on 
$5 \mathrm{~mL}$ swallows only, and not on either of the provocative tests. These subjects were less symptomatic, with median ES of 1 [interquartile range 1-2] compared to those with raised IRP on a provocative maneuver $(3[2-5] ; P<0.01)$. Further, none had a positive barium swallow or underwent invasive therapy. Conversely, 39 (32\%) had raised IRP on provocative testing but normal IRP on water swallows. Subjects who had raised IRP on provocative tests were similarly symptomatic whether or not this was associated with raised IRP on $5 \mathrm{~mL}$ swallows (median ES 3 for both groups; $P=0.55$ ).

\section{Structural EGJ00}

Determination of structural versus functional etiology could be made in 115 of 121 subjects (Figure 1). A definite structural cause of EGJOO was identified in 18 patients whereas the remaining 97 cases (84\%) were considered functional. Structural etiologies of EGJOO included mucosal stricture $(N=2)$, Schatzki ring $(N=2)$, dysfunction of fundoplication $(N=7)$, eosinophilic esophagitis $(N=2)$, gastric band $(N=1)$, sleeve gastrectomy $(N=1)$, and hiatal hernia with impingement at crural diaphragm $(N=3)$.

The symptomatology, barium esophagography and manometric characteristics of structural EGJOO did not differ significantly from the functional EGJOO cohort (Table 2). The number of opiate users was significantly higher among the functional group (29\% vs. $6 \%$ respectively; $P=0.04$ ).

\section{Functional EGJ00}

Clinical characteristics of functional EGJOO subjects are provided in Table 2. In total, 29/97 functional EGJOO subjects (30\%) with a baseline ES of 6 [5-8] underwent 
achalasia-type therapies (Figure 1). Primary therapy consisted of Botulinum toxin injection in 12, pneumatic dilatation in 16 and POEM in 1. Primary therapy was successful in 20/29 (69\%) subjects as assessed by short-term reduction in ES to $\leq 3$. Due to failed primary therapy, eight subjects proceeded to a secondary form of therapy (1 Botulinum toxin, 3 pneumatic dilatation, 4 POEM), of which 6 were successful. Therefore, following primary and secondary therapy, 26/29 (90\%) had short-term (3 month) treatment success ("responders"). At a median of 15 [8-22] months final follow up, the median ES remained low at 2 [0-2] with 23/29 (79\%) still in clinical remission. The procedural success rate by treatment modality was 10/13 (77\%), 13/19 (68\%), and 3/5 (60\%) for Botulinum toxin, pneumatic dilatation and POEM respectively $(P=0.79)$.

In 48 subjects (49\%), no achalasia-type therapy was provided (Figure 1); observation alone in 35 subjects and optimization of acid reducing therapy for reflux in 13 . Follow up was available in $32 / 48$ subjects (67\%) up to a duration of 9 [3.25 -19.5] months. At final follow up, $25 / 32(78 \%)$ subjects had a favorable symptomatic response (ES $\leq 3$ and reduction by $\geq 1$ point from baseline) despite no EGJ00-directed therapy having been administered ("spontaneous remitters").

Sixty-five subjects (67\%) with functional EGJOO had barium esophagography performed. Subjects who did not have esophagography performed were less symptomatic (median ES 2 vs. 4; $P<0.01$ ) and less severe findings on manometry (Supplementary Table S1). Of those who underwent esophagography, 19 (29\%) were "barium-positive." with evidence of holdup of contrast at the EGJ (35\% of timed barium studies positive vs. $24 \%$ positive on conventional barium; $P=0.41)$, whereas the remaining $47(72 \%)$ suggested clearance (barium-negative). Barium-positive patients were more likely to undergo achalasia-type 
therapy ( $83 \%$ vs. $27 \% ; P<0.001)$. Of the 15 barium-positive subjects who underwent therapy, $13 / 15$ were (87\%) were responders. However, 11 subjects who were bariumnegative nevertheless also underwent achalasia-type therapy, of whom 9 (82\%) were responders; i.e. the rate of therapeutic success was similar to that seen in barium-positive subjects $(P=1)$.

\section{Symptom-positive versus Symptom-negative Functional EGJ00}

Fifty-one functional EGJOO diagnoses were considered symptom-negative (eight had an alternative diagnosis to explain symptoms while 43 had a low symptom burden). The remaining 46 were considered symptom-positive EGJOO. Diagnostic variables in symptom-positive and symptom-negative EGJOO are provided in Table 3. The baseline IRP on both $5 \mathrm{~mL}$ swallows and RDC did not differ between groups. However, symptompositive EGJOO were more likely to have a raised IRP during solid swallows (93\% vs 57\% respectively; $P=0.00001)$. Furthermore, abnormal motility and pressurization patterns were more common during provocative tests than during $5 \mathrm{~mL}$ swallows (Figure 3 ), and were seen more frequently in symptom-positive EGJ00. Symptom reproduction during HRM provocative tests clearly discriminated between symptom-positive and -negative EGJOO, whereas there was no difference in the likelihood of symptoms being reproduced during $5 \mathrm{~mL}$ swallows between the two groups.

Twenty-nine symptom-positive EGJOO subjects underwent achalasia-type therapies, as described in the previous section. The remaining 17 symptom-positive EGJOO subjects did not undergo achalasia-type therapies due to comorbidities $(N=5)$, patient preference $(N=2)$ or because a strategy of opiate reduction was pursued instead $(N=3)$. The remaining $(N=7)$ were awaiting therapy or a decision for therapy. Symptom-positive 
EGJOO who had therapy were more symptomatic than those who did not (median ES 6 vs. 4 respectively; $P=0.01$; Supplementary Table S2).

\section{Accuracy of diagnostic modalities in functional EGJO0}

We compared diagnostic test performance characteristics using treatment responders $(N=26)$ as the reference standard for the presence of clinically relevant EGJOO, and spontaneous remitters $(N=25)$ as reference standard for the absence of disease. Therefore, the sample size for calculation of diagnostic accuracy of $5 \mathrm{~mL}$ water and provocative testing was 51 . However, 12 of these patients did not have barium esophagography performed, therefore the sample size for calculation of diagnostic accuracy in barium esophagography was 39. The sensitivity and specificity of manometric abnormalities found during provocative HRM tests for clinically relevant EGJOO was high at $85 \%$ and $84 \%$ respectively. The specificity did not significantly differ compared to $5 \mathrm{~mL}$ swallows and barium swallow; however, the sensitivity of provocative testing was significantly superior to $5 \mathrm{~mL}$ water $\mathrm{HRM}$ and barium esophagography, with sensitivity of $54 \%$ for both of the latter tests $(P<0.01$ and 0.02 for respective comparisons with provocative tests; Table 4). In particular, while barium swallow was highly specific for clinically relevant EGJOO, sensitivity was poor. Provocative HRM tests not only had a high positive predictive value for clinically relevant EGJOO but were superior to the other tests in ruling it out. The negative predictive value for provocative testing was $84 \%$; whereas the corresponding values for $5 \mathrm{~mL}$ HRM and barium esophagography was $64 \%$ and 58\% respectively. To account for the fact that not all patients had barium esophagography performed, we performed a sensitivity analysis excluding those patients. The diagnostic accuracy findings were not markedly different, and performance 
of provocative tests remained significantly superior to the other modalities (Supplementary Table S3).

The number of manometric tests with raised IRP (Figure 2) was not predictive of clinically relevant EGJOO. Both treatment responders and spontaneous remitters had a similar likelihood of having one test, two tests or three tests demonstrating raised IRP.

\section{Discussion}

We report findings from a large cohort of EGJOO subjects, using outcomes data in an effort to define diagnostic variables that can identify clinically relevant functional EGJ00. The most important findings were as follows. First, HRM findings during provocative testing correlate closely with clinical symptomatology. Secondly, barium swallow is highly specific, but appears to miss many cases of clinically relevant EGJO0. Third, provocative tests during HRM have a superior diagnostic accuracy for clinically relevant EGJOO compared with $5 \mathrm{~mL}$ swallows and barium swallow, being able to identify both those who will respond to therapy and those who spontaneously remit with a high degree of accuracy. Fourth, patients who had a raised IRP only on $5 \mathrm{~mL}$ swallows and not on provocative tests did not have significant disease requiring therapy.

We found a $69 \%$ primary therapeutic response in the select subset of functional EGJOO who underwent achalasia-type therapies, which rose to $90 \%$ following secondary treatment in initial non-responders. Therapeutic response rates have varied widely (between 29-100\%) in a number of small functional EGJOO cohorts, though little information was provided regarding method of patient selection for achalasia-type 
therapies. ${ }^{6,8,24-28}$ However, two recent studies reported 78-85\% initial response rates to achalasia-type therapies in functional EGJOO subjects who demonstrated barium stasis and/or impaired LES distensibility on FLIP. ${ }^{5,13}$ The rates of primary response seen in these latter two studies along with the present study are comparable to those in achalasia using Botulinum toxin, pneumatic dilatation and POEM, ${ }^{29-31}$ suggesting that a carefully selected subset of EGJOO does indeed represent a variant form of achalasia, and can be treated as such.

The key advance of our study is its demonstration of the value of provocative HRM tests in aiding patient selection for achalasia-type therapy. Abnormal findings on provocative testing were highly prevalent in those with significant symptoms but rarely found in EGJOO subjects who were minimally symptomatic or who had an alternate explanation for their symptoms (Table 3). In contrast, 5mL HRM was poor at discriminating such patients. Further, the presence of abnormal peristalsis, pressurization patterns or reproduction of typical symptoms during provocative testing identified clinically relevant EGJO0 with $85 \%$ sensitivity and $84 \%$ specificity, where clinical relevance was determined by natural history and therapeutic outcomes. The sensitivity in particular was significantly superior than that of $5 \mathrm{~mL}$ swallows and barium esophagography (54\% for both).

In other words, we found that barium esophagography missed many cases of clinically relevant EGJOO (11 of 24 treatment responders had normal barium emptying). This is consistent with findings of Triggs et al. who found that barium esophagography missed cases of EGJOO identified by FLIP that also went on to respond to achalasia-type therapy. ${ }^{13}$ The lack of barium holdup despite symptoms may reflect intact peristalsis 
facilitating esophageal emptying despite clinically relevant LES dysfunction. It is important to note however the variability in protocol, with both standard and timed barium studies performed, yet in any case there was no significant difference in the likelihood of demonstrating holdup based on the type of study performed. Also, it is not clear if the 1-minute holdup is as relevant as the more standard 5-minute measure used for achalasia-related obstruction, nor what minimum barium column height level should be considered relevant. However, we observed that unlike in achalasia, residual barium at 5 minutes was rarely observed; and even at 1 minute a tall column of barium $>5 \mathrm{~cm}$ was uncommon. Finally, not all patients had barium esophagography performed. The group who did not have barium performed tended to be less symptomatic with fewer manometric abnormalities (Supplementary Table S1). While this is a potential source of bias, a sensitivity analysis performed including only patients with esophagography performed did not show materially different results (Supplementary Table S3) and confirmed the superior diagnostic performance of provocative testing over the other modalities.

Regardless of variability in methodology, we found that a positive barium study had a $100 \%$ specificity for clinically relevant functional EGJOO that responds to therapy. For this reason, and the added utility that esophagography has in identifying structural causes of EGJOO, our findings should not be interpreted as completely diminishing the role of barium esophagography in the evaluation of these patients. Instead, our findings emphasize the value of incorporating multiple pieces of diagnostic information into the decision-making algorithm. For example, a symptomatic patient with functional EGJOO and pressurization or reproduction of symptoms during solid swallows should be considered for achalasia-type therapy even if the barium esophagogram shows no holdup 
at the EGJ. In contrast, the finding of raised IRP on $5 \mathrm{~mL}$ swallows but not during RDC or solids should be considered to indicate high likelihood of spontaneous symptom remission. By using complementary testing in such a fashion, unnecessary intervention can be avoided and conversely, patients who are likely to benefit from therapy can be positively identified. In specialized centers that have access to it, FLIP could conceivably be used as an additional complementary test to provide similar information, albeit at greater expense and with the requirement for sedation.

The study has limitations primarily relating to its retrospective design. Barium protocols were mixed, as described above. Treatment was not uniform, and modality of achalasiatype therapy was at the discretion of the physician and patient, reflecting real-world practice in this incompletely defined disease entity. Twenty percent of functional EGJOO subjects were lost to follow up, particularly those who were managed conservatively (Figure 1); so, it is possible that more of these patients developed delayed symptoms. However, this would not have materially affected our calculations of diagnostic accuracy as we only included patients with adequate follow up and used conservative thresholds for therapeutic success. Further, despite missing follow up, this remains one of the largest studies to date which includes treatment response in EGJOO. The use of natural history and therapeutic outcomes as a reference standard for disease has inherent limitations, however, its use as a surrogate in the absence of a true reference standard is accepted practice, ${ }^{32}$ and is validated by our corroborating findings in symptom-positive and symptom-negative patients. The use of the Eckardt score to define treatment response has several well-known limitations, ${ }^{33}$ and the definition of spontaneous remission as a reduction in Eckardt score of 1 point is arbitrary and not validated. Regardless, its use in 
outcome studies is common in achalasia, and it has also been used for this purpose in EGJO0.13

In summary, manometric findings from rapid drink challenge and solid swallows are highly accurate, and superior to barium esophagography in identifying clinically relevant functional EGJOO that responds to achalasia-type therapies. Given that provocative testing is simple and inexpensive to perform, it should be routinely added to the standard manometric protocol to increase the diagnostic accuracy of HRM.

\section{References}

1. Kahrilas PJ, Bredenoord AJ, Fox M, et al. The Chicago Classification of esophageal motility disorders, v3.0. Neurogastroenterology \& Motility 2015;27:160-174.

2. Richter JE, Clayton SB. Diagnosis and Management of Esophagogastric Junction Outflow Obstruction. American Journal of Gastroenterology 2019;114:544-547.

3. Babaei A, Lin EC, Szabo A, et al. Determinants of pressure drift in Manoscan ${ }^{\mathrm{TM}}$ esophageal high-resolution manometry system. Neurogastroenterology \& Motility 2015;27:277-284.

4. Babaei A, Szabo A, Yorio SD, et al. Pressure exposure and catheter impingement affect the recorded pressure in the Manoscan $360^{\mathrm{TM}}$ system. Neurogastroenterology \& Motility 2018;30.

5. Clayton SB, Shin CM, Ewing A, et al. Pneumatic dilation improves esophageal emptying and symptoms in patients with idiopathic esophago-gastric junction outflow obstruction. Neurogastroenterology \& Motility 2018. 
6. Lynch KL, Yang XY, Metz DC, et al. Clinical presentation and disease course of patients with esophagogastric junction outflow obstruction. Diseases of the Esophagus 2017;30:1-6.

7. Liu A, Woo M, Nasser Y, et al. Esophagogastric junction outflow obstruction on manometry: Outcomes and lack of benefit from CT and EUS. Neurogastroenterology \& Motility 2019;31.

8. DeLay K, Austin GL, Menard-Katcher P. Anatomic abnormalities are common potential explanations of manometric esophagogastric junction outflow obstruction. Neurogastroenterology \& Motility 2016;28:1166-1171.

9. Biasutto D, Mion F, Garros A, et al. Rapid drink challenge test during esophageal high resolution manometry in patients with esophago-gastric junction outflow obstruction. Neurogastroenterology \& Motility 2018;30.

10. Triggs JR, Carlson DA, Beveridge C, et al. Upright Integrated Relaxation Pressure Facilitates Characterization of Esophagogastric Junction Outflow Obstruction. Clinical Gastroenterology and Hepatology 2019.

11. Misselwitz B, Hollenstein M, Bütikofer S, et al. Prospective serial diagnostic study: the effects of position and provocative tests on the diagnosis of oesophageal motility disorders by high-resolution manometry. Alimentary Pharmacology \& Therapeutics 2020;51:706-718.

12. Clayton SB, Patel R, Richter JE. Functional and Anatomic Esophagogastic Junction Outflow Obstruction: Manometry, Timed Barium Esophagram Findings, and Treatment Outcomes. Clinical gastroenterology and hepatology 2016;14:907-911.

13. Triggs JR, Carlson DA, Beveridge C, et al. Functional luminal imaging probe panometry identifies achalasia-type esophagogastric junction outflow obstruction. Clinical Gastroenterology and Hepatology 2019. 
14. Sanagapalli S, Roman S, Hastier A, et al. Achalasia diagnosed despite normal integrated relaxation pressure responds favorably to therapy. Neurogastroenterology \& Motility 2019;31.

15. Wang YT, Tai LF, Yazaki E, et al. Investigation of Dysphagia After Antireflux Surgery by High-resolution Manometry: Impact of Multiple Water Swallows and a Solid Test Meal on Diagnosis, Management, and Clinical Outcome. Clinical gastroenterology and hepatology 2015;13:1575-1583.

16. Ang D, Hollenstein M, Misselwitz B, et al. Rapid Drink Challenge in high-resolution manometry: an adjunctive test for detection of esophageal motility disorders. Neurogastroenterology \& Motility 2016;29:e12902.

17. Ang D, Misselwitz B, Hollenstein M, et al. Diagnostic yield of high-resolution manometry with a solid test meal for clinically relevant, symptomatic oesophageal motility disorders: serial diagnostic study. The Lancet Gastroenterology \& hepatology 2017;2:654-661.

18. Sanagapalli S, Emmanuel A, Leong R, et al. Impaired motility in Barrett's esophagus: A study using high-resolution manometry with physiologic challenge. Neurogastroenterology \& Motility 2018;30:e13330.

19. Hollenstein $M$, Thwaites $\mathrm{P}$, Bütikofer S, et al. Pharyngeal swallowing and oesophageal motility during a solid meal test: a prospective study in healthy volunteers and patients with major motility disorders. Lancet Gastroenterol Hepatol 2017;2:644-653.

20. Sweis R, Anggiansah A, Wong T, et al. Normative values and inter-observer agreement for liquid and solid bolus swallows in upright and supine positions as assessed by esophageal high-resolution manometry. Neurogastroenterology \& Motility 2011;23:509. 
21. Sweis R, Anggiansah A, Wong T, et al. Assessment of esophageal dysfunction and symptoms during and after a standardized test meal: development and clinical validation of a new methodology utilizing high-resolution manometry. Neurogastroenterology \& Motility 2014;26:215-228.

22. de Oliveira JM, Birgisson S, Doinoff C, et al. Timed barium swallow: a simple technique for evaluating esophageal emptying in patients with achalasia. American journal of roentgenology 1997;169:473-479.

23. Moonen A, Annese V, Belmans A, et al. Long-term results of the European achalasia trial: a multicentre randomised controlled trial comparing pneumatic dilation versus laparoscopic Heller myotomy. Gut 2016;65:732-739.

24. Scherer JR, Kwiatek MA, Soper NJ, et al. Functional Esophagogastric Junction Obstruction with Intact Peristalsis: A Heterogeneous Syndrome Sometimes Akin to Achalasia. Journal of Gastrointestinal Surgery 2009;13:2219-2225.

25. van Hoeij FB, Smout A, Bredenoord AJ. Characterization of idiopathic esophagogastric junction outflow obstruction. Neurogastroenterology \& Motility $2015 ; 27: 1310-1316$.

26. Okeke FC, Raja S, Lynch $\mathrm{KL}$, et al. What is the clinical significance of esophagogastric junction outflow obstruction? evaluation of 60 patients at a tertiary referral center. Neurogastroenterology \& Motility 2017;29.

27. Pérez-Fernández MT, Santander C, Marinero A, et al. Characterization and followup of esophagogastric junction outflow obstruction detected by high resolution manometry. Neurogastroenterology \& Motility 2016;28:116-126.

28. Schupack D, Katzka DA, Geno DM, et al. The clinical significance of esophagogastric junction outflow obstruction and hypercontractile esophagus in high resolution esophageal manometry. Neurogastroenterology \&amp; Motility 2017;29:1-9. 
29. Vaezi MF, Richter JE, Wilcox CM, et al. Botulinum toxin versus pneumatic dilatation in the treatment of achalasia: a randomised trial. Gut 1999;44:231.

30. Boeckxstaens GE, Annese V, des Varannes S, et al. Pneumatic Dilation versus Laparoscopic Heller's Myotomy for Idiopathic Achalasia. The New England Journal of Medicine 2011;364:1807-1816.

31. Ponds FA, Fockens P, Lei A, et al. Effect of Peroral Endoscopic Myotomy vs Pneumatic Dilation on Symptom Severity and Treatment Outcomes Among Treatment-Naive Patients With Achalasia. JAMA 2019;322:134-144.

32. Reitsma JB, Rutjes A, Khan KS, et al. A review of solutions for diagnostic accuracy studies with an imperfect or missing reference standard. Journal of Clinical Epidemiology 2009;62:797-806.

33. Sanagapalli S, Sweis R. Achalasia: It Is Not All Black and White. Current Gastroenterology Reports 2017;19:27. 
Table 1: Baseline characteristics

\begin{tabular}{|c|c|}
\hline \multicolumn{2}{|l|}{ Clinical characteristics } \\
\hline$N$ & 121 \\
\hline Age, years & $57.5 \pm 15.6$ \\
\hline Males & $46(38 \%)$ \\
\hline Proton pump inhibitor use & $81(67 \%)$ \\
\hline Opiate use & $29(24 \%)$ \\
\hline Eckardt score & $3[2-5]$ \\
\hline Dysphagia & $92(76 \%)$ \\
\hline At least daily dysphagia & $61(50 \%)$ \\
\hline Barium holdup & $21 / 83(25 \%)^{*}$ \\
\hline \multicolumn{2}{|l|}{ Manometric characteristics } \\
\hline $5 \mathrm{~mL}$ IRP, $\mathrm{mmHg}$ & $18.8 \pm 7.7$ \\
\hline RDC IRP, mmHg & $12.0 \pm 9.4$ \\
\hline \multicolumn{2}{|l|}{ Abnormal pressurization } \\
\hline $5 \mathrm{~mL}$ water & $4 \%$ \\
\hline Provocative tests & $18 \%$ \\
\hline \multicolumn{2}{|l|}{ Abnormal motility } \\
\hline $5 \mathrm{~mL}$ water & $28 \%$ \\
\hline Provocative tests & $41 \%$ \\
\hline \multicolumn{2}{|l|}{ Symptoms reproduced } \\
\hline $5 \mathrm{~mL}$ water & $4 \%$ \\
\hline Provocative tests & $32 \%$ \\
\hline
\end{tabular}

* 36 timed and 47 conventional barium swallows performed 
Table 2: Characteristics of structural and functional EGJOO

\begin{tabular}{|c|c|c|c|}
\hline & $\begin{array}{l}\text { Structural } \\
\mathrm{N}=18\end{array}$ & $\begin{array}{l}\text { Functional } \\
\mathrm{N}=97\end{array}$ & $P$ \\
\hline $5 \mathrm{~mL}$ IRP, $\mathrm{mmHg}$ & 16 & 19.5 & 0.10 \\
\hline RDC IRP & 10.5 & 12.5 & 0.40 \\
\hline $\begin{array}{l}\text { Raised IRP solid } \\
\text { swallows }\end{array}$ & 15/18 (83\%) & $68 / 92(74 \%)$ & 0.55 \\
\hline Opiate use & $1 / 18(6 \%)$ & $28 / 97(29 \%)$ & 0.04 \\
\hline $\begin{array}{l}\text { Proton pump } \\
\text { inhibitor use }\end{array}$ & 13/18 (72\%) & $62 / 97(64 \%)$ & 0.60 \\
\hline Barium holdup & $2 / 14(14 \%)$ & 19/65 (29\%) & 0.30 \\
\hline Baseline ES & $4.5[3-5.75]$ & $3[2-5]$ & 0.08 \\
\hline
\end{tabular}


Table 3: Symptom-negative versus symptom-positive EGJOO

\begin{tabular}{|c|c|c|}
\hline & $\begin{array}{l}\text { Symptom-negative EGJOO } \\
\mathrm{N}=51\end{array}$ & $\begin{array}{l}\text { Symptom-positive } \\
\text { EGJO0 } \\
\mathrm{N}=46\end{array}$ \\
\hline Eckardt score & $2[1-3]$ & $5[4-7]^{* * *}$ \\
\hline $5 \mathrm{~mL}$ IRP & $19.0 \pm 7.4$ & $20.0 \pm 7.6$ \\
\hline Raised 5mL IRP & $76 \%$ & $67 \%$ \\
\hline RDC IRP & $11.4 \pm 6.8$ & $13.8 \pm 12.0$ \\
\hline Raised RDC IRP & $68 \%$ & $65 \%$ \\
\hline Raised IRP solid swallows & $57 \%$ & $93 \% * * *$ \\
\hline $\begin{array}{l}\text { Abnormal pressurization on } 5 \mathrm{~mL} \\
\text { swallows }\end{array}$ & $2 \%$ & $7 \%$ \\
\hline $\begin{array}{l}\text { Abnormal pressurization on } \\
\text { provocative tests }\end{array}$ & $6 \%$ & $26 \% *$ \\
\hline $\begin{array}{l}\text { Abnormal motility pattern on } \\
5 \mathrm{~mL} \text { swallows }\end{array}$ & $16 \%$ & $48 \% * *$ \\
\hline $\begin{array}{l}\text { Abnormal motility pattern solid } \\
\text { swallows }\end{array}$ & $16 \%$ & $65 \% * * *$ \\
\hline $\begin{array}{l}\text { Symptoms reproduced during } \\
5 \mathrm{~mL} \text { swallows }\end{array}$ & $2 \%$ & $9 \%$ \\
\hline $\begin{array}{l}\text { Symptoms reproduced during } \\
\text { provocative tests }\end{array}$ & $6 \%$ & $61 \% * * *$ \\
\hline Barium holdup & $1 / 26(4 \%)$ & $18 / 39(46 \%)^{* * *}$ \\
\hline
\end{tabular}

${ }^{*} P<0.01 ;{ }^{* *} P<0.001 ;{ }^{* * *} P<0.0001$ 
Table 4: Diagnostic accuracy for clinically relevant functional EGJOO

\begin{tabular}{|c|c|c|c|c|c|c|}
\hline & \multicolumn{3}{|c|}{ Sensitivity } & \multicolumn{3}{|c|}{ Specificity } \\
\hline & $\%$ & $\begin{array}{l}\text { Difference with } \\
\text { provocative } \\
\text { tests }(95 \% \mathrm{CI})\end{array}$ & $P^{*}$ & $\%$ & $\begin{array}{l}\text { Difference with } \\
\text { provocative } \\
\text { tests }(95 \% \mathrm{CI})\end{array}$ & $P^{*}$ \\
\hline Provocative tests & $\begin{array}{l}22 / 26 \\
(85 \%)\end{array}$ & - & - & $\begin{array}{l}21 / 25 \\
(84 \%)\end{array}$ & - & - \\
\hline 5mL swallows & $\begin{array}{l}14 / 26 \\
(54 \%)\end{array}$ & $\begin{array}{l}31 \% \quad(13 \text { to } \\
49 \%)\end{array}$ & $<0.01$ & $\begin{array}{l}21 / 25 \\
(84 \%)\end{array}$ & $0 \%(-16$ to $16 \%)$ & 1 \\
\hline $\begin{array}{l}\text { Barium } \\
\text { esophagography }\end{array}$ & $\begin{array}{l}13 / 24 \\
(54 \%)\end{array}$ & $29 \%(8$ to $51 \%)$ & 0.02 & $\begin{array}{l}15 / 15 \\
(100 \%)\end{array}$ & $\begin{array}{lll}-20 \% \quad(-40 \text { to } \\
1 \%)\end{array}$ & 0.08 \\
\hline
\end{tabular}

${ }^{*} P$ value for comparison with provocative test using McNemar's test 
Figure 1: Patient flow 
Figure 2: Subjects with raised IRP by HRM modality 
Figure 3: Added value of provocative tests in EGJ00. 5mL swallows in A-E (left column) all demonstrated normal peristalsis and no abnormal pressurization. However, provocative tests (right column) demonstrated: A, esophageal body pressurization during RDC; $\mathrm{B}$, hypercontractile peristalsis; C, compartmentalized pressurization; D, simultaneous contraction in distal esophagus; E, pan-esophageal pressurization. All esophageal topographic plots displayed with 30mmHg black isobaric contour line. 